\title{
Threat perception predicts cognitive behavioral therapy outcomes in anxious children
}

\author{
Katharina Manassis ${ }^{1}$, Kathryn M. Hum ${ }^{2}$, Trevor Changgun Lee ${ }^{1}$, Gloria Zhang ${ }^{1}$, Marc D. Lewis ${ }^{3}$ \\ ${ }^{1}$ Department of Psychiatry, Hospital for Sick Children, University of Toronto, Toronto, Canada \\ ${ }^{2}$ Department of Human Development and Applied Psychology, University of Toronto, Toronto, Canada \\ ${ }^{3}$ Behavioral Science Institute, Radboud University, Nijmegen, The Netherlands \\ Email: katharina.manassis@sickkids.ca
}

Received 9 January 2013; revised 7 February 2013; accepted 17 February 2013

\begin{abstract}
Objective: Anxiety disorders of childhood are prevalent, debilitating conditions that do not always respond to existing treatments. Attentional biases towards threatening stimuli have been reported in anxious children and hypothesized to interfere with treatment response. Therefore, we examined such biases in children with anxiety disorders in relation to cognitive behavioral therapy (CBT) outcomes. Method: Thirty-eight children diagnosed with anxiety disorders in a specialized clinic ( 21 girls and 17 boys; age = $10.50 \pm 1.11$ years) and 36 unaffected community controls (19 girls and 17 boys; age $=10.20 \pm 1.07$ years) participated. Participants completed standardized questionnaire measures of anxiety and a probe position task (PPT) with facial cues. This task often reveals a response slowing effect related to threatening faces in vulnerable individuals. Children with anxiety disorders repeated these measures after completing CBT. Results: Groups did not differ in performance on the PPT, but angry/calm incongruent difference scores were significantly associated with self-reports of social anxiety and state anxiety. When controlling for pre-CBT anxiety levels, incongruent difference scores involving angry faces predicted post-CBT anxiety disorders index scores on the Multidimensional Anxiety Scale for Children. PPT scores did not change significantly with CBT. Conclusions: Attentional bias towards threat on the PPT task may predict response to CBT and appears linked to social anxiety. Interventions to ameliorate this bias merit further study, as they might improve treatment outcomes for anxious, especially socially anxious, children.
\end{abstract}

Keywords: Anxiety Disorder; Children; Threat Perception; Emotion; Cognitive Behavioral Therapy

*This study was funded by a Type A Grant of the Ontario Mental Health Foundation.

\section{INTRODUCTION}

Childhood anxiety disorders are prevalent, debilitating, and associated with adverse long term outcomes [1,2]. Evidence-based treatments include cognitive behavioral therapy (CBT) and serotonin-specific medications. Recent evidence suggests that their combination may be more effective than either alone $[3,4]$. Nevertheless, substantial numbers of affected children fail to respond, or suffer adverse effects from medication [4,5].

Existing psychological treatments focus on developing children's coping strategies, parenting strategies, or both [1]. They do not address the tendency for some children to become anxious more frequently than others in response to environmental stimuli. This tendency has been linked to undue attention to threat in anxious children [6, 7]. Based on studies to date, it is unclear whether or not this vulnerability is ameliorated by $\mathrm{CBT}$, or predicts a less robust treatment response. If the latter were true, adjunctive interventions targeting threat perception might enhance outcomes. Therefore, the relationship between anxiety-focused CBT and threat perception in children merits clarification.

Relevant background pertains to the nature of threat perception, evidence for disorder-specificity, and existing studies examining threat perception in relation to CBT. Attention to threat has been found consistently in anxious individuals of different ages [8], but with evidence that state anxiety (i.e. anxiety in the experimental situation) may need to be elevated for some anxious individuals to display threat-biased attention [9]. Experimental induction of threat-biased attention in nonanxious individuals increased their vulnerability to emotional responses on standardized stress tasks [10], supporting amelioration of such bias as a therapeutic goal. Tasks that measure attention to threat vary in the degree to which the threat is explicit (i.e. amenable to conscious interpretations and responses) versus implicit [8]. For example, common explicit tasks require the interpretation of threat-ambiguous stimuli; common implicit tasks meas- 
ure changes in performance after very brief exposure to threatening stimuli. Neuroimaging studies suggest that common psychological interventions influence attention via activity in the lateral prefrontal cortex [11], suggesting that performance on tasks involving explicit rather than implicit attention to threat would be more likely to change in response to CBT.

Biased attention to threat may be disorder-specific [6, 7]. For example, Spector, Pecknold and Libman [12] found a specific bias to social threat in adults with generalized Social Phobia. Waters and colleagues [13] found that, in children with Generalized Anxiety Disorder, only severe anxiety or the presence of social phobia was linked to attentional bias to threat on a visual probe task using faces. Similarly, Perez-Edgar and colleagues [14] found that behaviorally inhibited preschoolers (vulnerable to social anxiety) showed more stable forms of social withdrawal if they oriented towards threats in a dot-probe task than if they did not. Complicating these diagnostic specificity findings, however, is the high degree of comorbidity found among anxiety disorders in prepubertal children [1].

In a recent review of threat perception in CBT with anxious adults, 10 of 13 studies showed reduced attentional bias after CBT [15]. However, included studies used a variety of more or less explicit experimental paradigms. In anxious children, Legerstee and colleagues [16] found that difficulty disengaging from threatening pictures on a dot probe (implicit) task was associated with treatment nonresponse to CBT. Subsequently, they linked normalization of this bias to child response to "stepped care CBT" (an unusually intensive child- and parentfocused intervention) [17]. However Waters and colleagues [18] found that threat interpretation bias on stories (an explicit task) responded to a standard child CBT protocol, but bias on a visual probe task (an implicit task) persisted.

In the present study, we examined attention to threat in children with anxiety disorders and non-anxious controls on a Probe Position Task (PPT) using facial cues [19], an implicit task based on the dot probe paradigm. Children with anxiety disorders repeated the task after twelve sessions of CBT. Given the literature reviewed above, we hypothesized that:

- Clinically anxious children would show greater attention to threat (i.e. differential response to angry versus calm or happy faces) on the PPT than normal controls;

- Given that task stimuli were facial (i.e., social cues) and the literature reviewed regarding social anxiety, difference scores would correlate with self-reported social anxiety;

- Difference scores would predict response to CBT on a self-report measure of trait anxiety;

- As the PPT is an implicit rather than explicit task, difference scores would not change significantly in response to CBT.

\section{METHOD}

\subsection{Participants}

Ethical approval of the study was obtained from the authors' university and the children's hospital that referred participants. Parents provided informed consent and children provided assent.

Fifty-five children, 8 - 12 years of age, enrolled in a 12-week group-based cognitive behavioral therapy program ("Coping Bear"; a previously evaluated adaptation of Kendall's Coping Cat program [20,21]) were referred from a specialized outpatient anxiety disorders clinic at a tertiary care children's hospital to participate in the study. The clinic serves a large urban and suburban population. Diagnoses had been verified using semi-structured parent and child interviews (Anxiety Disorders Interview Schedule-ADIS [22]). All ADIS interviewers were child psychologists or child psychiatrists trained to reliability, with at least 3 years experience using it in research. Children had a primary, most impairing diagnosis of an Axis I anxiety disorder (Generalized Anxiety Disorder, Social Anxiety Disorder or Separation Anxiety Disorder). Generalized Anxiety Disorder was the most common diagnosis, and $40 \%$ of children met criteria for more than one anxiety diagnosis, consistent with previous research samples from this clinic. Forty-seven families of eligible children consented to have their children participate and all completed treatment. For inclusion in the present analysis, however, the child had to complete a research assessment at pre-treatment and at post- treatment, less than two months following the end of therapy, yielding a final sample of 38 clinical children. These did not differ significantly in age, gender, or parental education level from the original 55 referred. There were 21 girls and 17 boys, with a mean age of 10.50 years +1.11 years.

Forty-one age-matched children were recruited from the community to serve as a normative comparison group. They were required to rate within the normal range by parent report on the Child Behavior Checklist (CBCL) Internalizing scale (T-score $<60)$ [23], resulting in five exclusions and yielding a final sample of 36 . There were 19 girls and 17 boys, with a mean age of 10.20 years + 1.07 years. Clinical and comparison children did not differ significantly in age, gender, or parental education level. About two thirds of parents in both groups had some university education, and ethnicity was $75 \%$ Caucasian in both groups.

\subsection{Procedure}

The Probe Position Task was the key experimental measure. Questionnaires included the Multidimensional Anxiety Scale for Children (MASC) [24], the State-Trait 
Anxiety Inventory for Children (STAIC-S) [25], the Child Behavior Checklist (CBCL) [23] by parent report, and a parent report of basic demographic information using a questionnaire from the Ontario Child Health Study (OCHS) [26]. Administration of measures was counterbalanced to minimize order effects. All children completed measures in a single research visit initially, and children in the clinical group repeated their measures in a single visit after CBT.

Probe Position Task (PPT): The child was seated in front of a computer monitor, with the distance and alignment to the monitor controlled by a chin rest. Parents waited in an adjacent room while a research assistant sat next to the child and provided scripted task instructions and on-line assistance if necessary. Participants were instructed to make responses by clicking one of two buttons on a response pad using either index finger. They were given a practice block of 12 trials to ensure proficiency with the task, with the opportunity to repeat the practice block if needed.

The PPT used emotional faces from the NimStim set of facial expressions (see below). The task was modified from Mogg and colleagues [19] and is illustrated in Figure 1. Each trial began with a central fixation cross appearing for $300 \mathrm{~ms}$ with a grey box located to the left and right of the cross. A face cue then appeared in either the left or right grey box for 500 milliseconds. Fifty milliseconds after the face disappeared, a $1 \mathrm{~cm}$ white dot was presented in either the left or right grey box. This stimulus remained on-screen until a response was made or until 1 second had elapsed. Participants were asked to press one of two buttons, using the index finger of each hand, to indicate as quickly and as accurately as possible on which side the white dot was presented. Each face cue and target appeared equally often in left and right locations. The locations of the face cue and target were congruent on $75 \%$ of trials (i.e., the target appeared in the same spatial location as the face cue) and incongruent on $25 \%$ of trials (i.e., the target appeared in the opposite location to the face cue). Participants were asked to keep their gaze focused on the central fixation position throughout the task. They were informed that the target was more likely to appear in the location of the face cue, and they would win points for correct responses and lose points for incorrect responses. Data obtained from this task usually reflect a response slowing effect related to the emotion valence of the face. This effect is particularly salient in incongruent trials, which is thought to relate to subjects' difficulty disengaging attention from the threat location [19].

Response time difference scores were calculated separately for congruent and incongruent trials by subtracting the average participant calm face response times from the average angry face response times, the average participant happy face response times from the average an-

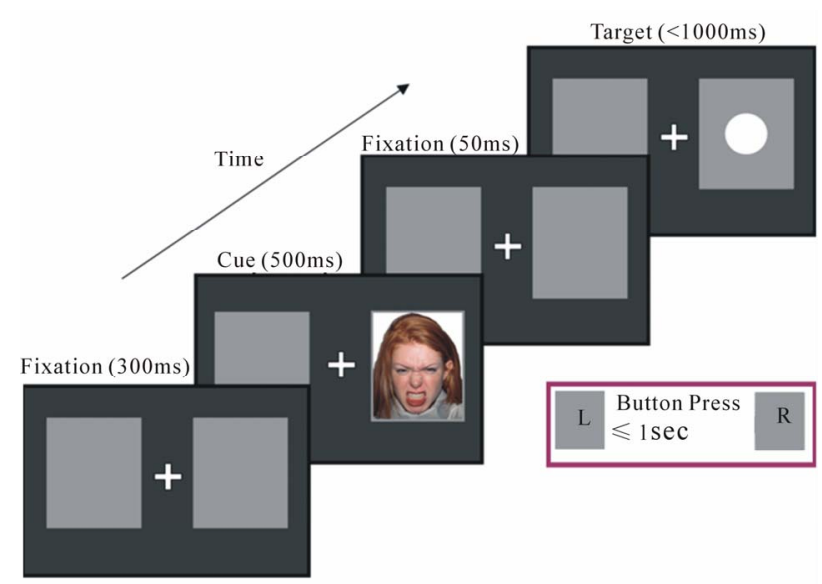

(a)

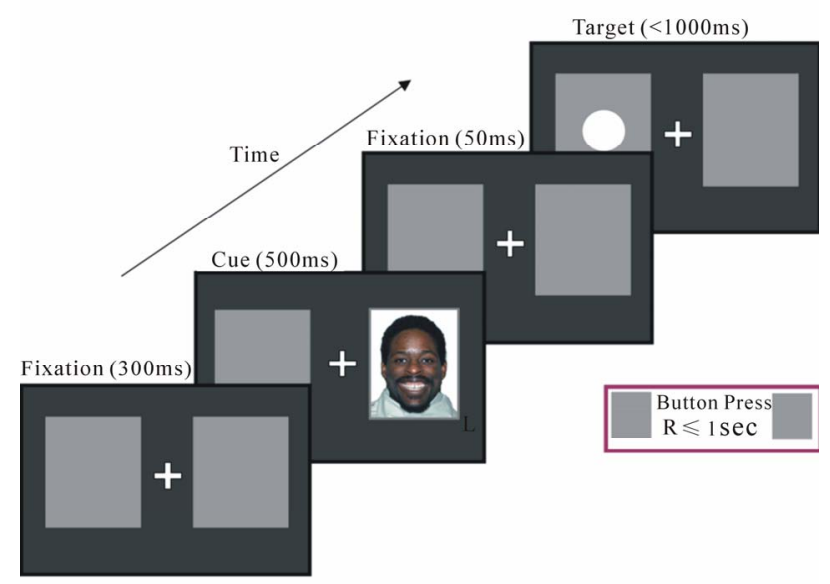

(b)

Figure 1. (a) Probe position task: congruent trial; (b) Probe position task: incongruent trial.

gry face response times, and the average calm face response times from the average happy face response times. Positive scores mean that participants responded more slowly to the first emotion (for example, angry in angry/calm difference score), while negative scores mean that participants responded more quickly to the first emotion.

Task stimuli were taken from the NimStim set of facial expressions [27]. This set contains a large number of color photographs of multi-racial actors posing a variety of facial expressions. Angry, happy, and calm emotion faces were used, with open and closed mouth conditions, posed by three models of each gender. Angry faces served as the threatening or negative emotion valence condition, calm faces (i.e., a slightly pleased or serene expression) as the comparison condition, and happy faces as the positive emotion valence condition. To ensure children's recognition of emotional valence, models with ambiguous emotional expressions were removed.

Questionnaires: The Multidimensional Anxiety Scale 
for Children (MASC [24]) is a 39-item self-report measure evaluating anxious symptoms in youth aged 8 to 19 years. A total T-score is obtained based upon the child's gender and age. Scaled T-scores are also attained for four factors (i.e., Physical Symptoms, Social Anxiety, Harm Avoidance, and Separation/Panic) and 2 indices (i.e., Anxiety Disorder (ADI) and Inconsistency Index). The MASC has good internal reliability, test-retest reliability, and discriminant validity. The MASC was used as the main indicator of trait anxiety in children.

The State-Trait Anxiety Inventory for Children (State version) (STAIC [25]) measures how anxious a child is at one particular moment (i.e., just prior to the experimental task). It is a 20-item self-report measure, which uses a 3-point scale (almost never, sometimes, always). It has been extensively normed and validated, and was used as the main indicator of state anxiety.

The Family and Household Form from the Ontario Child Health Study (OCHS [26]) provides basic demographic information in a systematic fashion based on parent report.

The Child Behavior Checklist (CBCL [23]) is a standardized, 118-item assessment of social competence and behavior problems in children 4 to 18 years, with separate internalizing and externalizing domains. It has been extensively normed and validated, with high internal and test-retest reliability (0.6 to 0.9 for various subscales).

Cognitive Behavioral Therapy: "Coping Bear" is a manualized program consisting of 12 weekly 1.5 hour sessions for children with anxiety disorders (6 to 8 children per group) with concurrent group sessions for parents (at least one parent per child attends consistently). Child sessions focus on recognizing anxious feelings and coping with them using relaxation, adaptive self-talk, and problem-solving. Exposure to feared situations is then encouraged. Parent sessions focus on supporting children's use of new coping strategies and problem-solving of common parenting challenges, based on the book "Keys to Parenting Your Anxious Child, 2nd Edition" [28]. Parents and children were assigned homework to practice new skills between sessions.

Statistical Procedures: Preliminary group comparisons were done on measures of anxiety using t-tests (with Bonferroni correction for number of comparisons), and on rates of valid trials. Group differences in PPT scores were examined using $2 \times 2$ ANOVAs with group (clinical and nonclinical) and facial emotion cues. As each contrast between emotions reflects a different type of bias, emotions were examined in pairs. Differences in mean response times between emotions were calculated (PPT difference scores), and bivariate correlations examined associations between anxiety measures and these scores. Regression analyses were used to test the capacity for PPT difference scores to predict change in anxiety meas- ures with CBT in clinical participants. Finally, two-way repeated measures ANOVAs examined differential changes in PPT scores by emotion with CBT, for the anxious group as a whole and then for treatment responders only.

\section{RESULTS}

\subsection{Preliminary Analyses}

Table 1 shows the means and standard deviations for all anxiety measures by group, confirming higher anxiety in the clinical than the control group by both parent and child report. Because children with anxiety disorders attended the clinic based on parent referral, not all of them had high self-reports of anxiety pre-treatment. Improved recognition of anxiety with CBT in some children likely accounted for the lack of group differences in anxiety self-reports from pre- to post-treatment. Groups did not differ significantly in their rates of valid trials (responses made between 200 and 1000 msec post-target (dot) presentation).

Mean PPT scores are shown by clinical status in Table 1. Assumptions of normality were examined for all key variables and were met. We examined potential associations between PPT difference scores and age, gender, and $\mathrm{CBCL}$ externalizing scores in order to control for these

Table 1. Mean anxiety scale and probe position task scores by clinical status.

\begin{tabular}{|c|c|c|c|}
\hline Scale (t scores): & $\begin{array}{c}\text { Anxiety } \\
\text { Disorder } \\
\text { Pre-CBT } \\
(\mathrm{n}=38) \\
\text { Mean (SD) }\end{array}$ & $\begin{array}{c}\text { Controls } \\
\text { Pre-CBT } \\
(\mathrm{n}=36) \\
\text { Mean (SD) }\end{array}$ & $\begin{array}{c}\text { Anxiety } \\
\text { Disorder } \\
\text { Post-CBT } \\
(\mathrm{n}=38) \\
\text { Mean (SD) }\end{array}$ \\
\hline MASC Total & $55.53(9.33)$ & $48.64(7.71)^{1}$ & $54.67(10.24)$ \\
\hline MASC ADI & $54.62(8.56)$ & $48.03(8.49)^{1}$ & $54.26(9.72)$ \\
\hline MASC Social Anxiety & $52.68(11.80)$ & $49.22(9.76)$ & $52.55(11.00)$ \\
\hline STAIC Total & $50.43(10.57)$ & $43.33(10.06)^{1}$ & ${ }^{1} 44.14(9.83)$ \\
\hline CBCL Internalizing & $67.28(9.83)$ & $47.97(8.98)^{1}$ & $61.67(10.37)$ \\
\hline
\end{tabular}

PPT Response Time (ms):

$\begin{array}{cccc}\text { PPT Congruent Angry } & 361(47) & 376(61) & 322(28) \\ \text { PPT Congruent Calm } & 355(48) & 375(59) & 326(35) \\ \text { PPT Congruent Happy } & 355(48) & 372(63) & 324(34) \\ \text { PPT Incongruent Angry } & 383(63) & 410(81) & 364(42) \\ \text { PPT Incongruent Calm } & 386(54) & 412(79) & 373(41) \\ \text { PPT Incongruent Happy } & 389(56) & 416(79) & 365(47)\end{array}$

MASC $=$ Multidimensional Anxiety Scale for Children; ADI $=$ Anxiety Disorders Index; STAIC = State-Trait Anxiety Inventory for Children (state version); $\mathrm{CBCL}=$ Child Behavior Checklist; PPT $=$ Probe Position Task; ${ }^{1}$ Significant group differences pre-CBT (all were $\mathrm{p}<0.001$ uncorrected, and $\mathrm{p}<0.005$ when corrected for number of comparisons). 
in subsequent analyses if needed, but none were found.

\subsection{Examining Group Differences in Attention to Threat on PPT}

Two by two ANOVAs showed no group by emotion cue interactions for the six pairs of emotions examined: congruent angry/calm $(\mathrm{F}(1.71)=0.11 ; \mathrm{p}=0.74)$, congruent angry/happy $(\mathrm{F}(1.71)=0.67 ; \mathrm{p}=0.41)$, congruent happy/calm $(\mathrm{F}(1.71)=0.24 ; \mathrm{p}=0.62)$, incongruent angry/calm $(\mathrm{F}(1.71)=0.02 ; \mathrm{p}=0.88)$, incongruent angry/happy $(\mathrm{F}(1.71)=0.02 ; \mathrm{p}=0.89)$, incongruent happy/ $\operatorname{calm}(\mathrm{F}(1.71)=0.07 ; \mathrm{p}=0.77)$.

\subsection{Anxiety, Social Anxiety, PPT Difference Scores}

PPT difference scores were calculated for angry versus calm and angry versus happy, to compare stimuli of negative emotional valence with nonemotional and positive valence stimuli, respectively. Difference scores for happy versus calm were included to ensure that findings were not merely related to emotional arousal rather than threat. Difference scores were not correlated with total anxiety reported on the MASC or the CBCL for the sample as a whole (see Table 2). However, difference scores for angry/calm and angry/happy incongruent trials were correlated with the Social Anxiety subscale of the MASC, albeit at trend level for angry/happy. STAIC scores (i.e., state anxiety) were also significantly correlated with these incongruent PPT scores. When using a partial correlation to control for STAIC scores, the level of significance of the association between Social Anxiety and these incongruent PPT difference scores remained unchanged suggesting that the association cannot be attributed entirely to state anxiety.

\subsection{PPT Difference Scores and CBT Response}

For self-reported treatment response (anxious group only, $\mathrm{n}=38$ ), we examined the Anxiety Disorders Index (ADI) of the MASC, as we were interested in changes in the most clinically relevant anxiety symptoms reported, and for parent-reported treatment response we examined the Internalizing Score of the CBCL. Regression analyses were performed with the post-treatment values of these measures serving as dependent variables. Pre-treatment values for each measure were entered first, and then each PPT difference score was entered to determine its capacity to predict change. The post-treatment ADI was predicted by the incongruent angry versus calm difference score in addition to the ADI pre-value, and by the incongruent angry versus happy difference score in addition to the ADI pre-value (see Table 3), suggesting that slower response times for angry versus calm/happy faces for incongruent trials pre-treatment predicted greater anxi-
Table 2. Bivariate correlations ( $\mathrm{R}$ values) between anxiety, age, and PPT difference scores.

\begin{tabular}{ccccccc}
\hline $\begin{array}{c}\text { Variables } \\
(\mathrm{N}=74)\end{array}$ & MAS & MAS & $\begin{array}{c}\text { MAS } \\
\text { ADI }\end{array}$ & $\begin{array}{c}\text { Soc. } \\
\text { STAI }\end{array}$ & $\begin{array}{c}\text { CBCL } \\
\text { Int. }\end{array}$ & Age \\
\hline PPT Con. & -0.12 & -0.09 & -0.10 & 0.07 & 0.02 & -0.11 \\
AN/CA & $\mathrm{p}=0.30$ & $\mathrm{p}=0.43$ & $\mathrm{p}=0.40$ & $\mathrm{p}=0.54$ & $\mathrm{p}=0.87$ & $\mathrm{p}=0.43$ \\
PPT Con. & -0.09 & -0.08 & -0.15 & -0.06 & -0.09 & -0.15 \\
AN/HA & $\mathrm{p}=0.43$ & $\mathrm{p}=0.49$ & $\mathrm{p}=0.20$ & $\mathrm{p}=0.62$ & $\mathrm{p}=0.41$ & $\mathrm{p}=0.27$ \\
PPT Con. & 0.02 & 0.04 & 0.10 & 0.15 & 0.12 & 0.03 \\
HA/CA & $\mathrm{p}=0.89$ & $\mathrm{p}=0.76$ & $\mathrm{p}=0.40$ & $\mathrm{p}=0.19$ & $\mathrm{p}=0.31$ & $\mathrm{p}=0.81$ \\
PPT Inc. & 0.08 & 0.14 & 0.30 & 0.23 & 0.01 & -0.19 \\
AN/CA & $\mathrm{p}=0.49$ & $\mathrm{p}=0.23$ & $\mathrm{p}=0.01$ & $\mathrm{p}=0.04$ & $\mathrm{p}=0.91$ & $\mathrm{p}=0.17$ \\
PPT Inc. & 0.10 & 0.12 & 0.20 & 0.22 & -0.06 & -0.06 \\
AN/HA & $\mathrm{p}=0.40$ & $\mathrm{p}=0.30$ & $\mathrm{p}=0.07$ & $\mathrm{p}=0.05$ & $\mathrm{p}=0.62$ & $\mathrm{p}=0.65$ \\
PPT Inc. & 0.06 & 0.05 & -0.06 & 0.05 & 0.07 & -0.18 \\
HA/CA & $\mathrm{p}=0.60$ & $\mathrm{p}=0.68$ & $\mathrm{p}=0.59$ & $\mathrm{p}=0.69$ & $\mathrm{p}=0.57$ & $\mathrm{p}=0.20$ \\
\hline
\end{tabular}

$\mathrm{PPT}=$ Probe Position Task; MAS $=$ Multidimensional Anxiety Scale for Children; ADI $=$ Anxiety Disorders Index; Soc. $=$ Social Anxiety; STAI $=$ Stat-Trait Anxiety Inventory for Children (state version); CBCL Int. = Child Behavior Checklist Internalizing Score; Con. AN/CA = Congruent AngryCalm; Con. AN/HA = Congruent Angry-Happy; Inc. AN/CA = Incongruent Angry-Calm; Inc. AN/HA = Incongruent Angry-Happy.

Table 3. Two significant regression analyses predicting posttreatment anxiety disorders index in anxious children.

\begin{tabular}{cccccc}
\hline Variable Entered & $\begin{array}{c}\text { Unstand. } \\
\text { Coeff. }\end{array}$ & $\begin{array}{c}\text { Stand. } \\
\text { Coeff. (Beta) }\end{array}$ & $\mathrm{t}$ & $\mathrm{p}$ & $\begin{array}{c}\mathrm{F}, \mathrm{R}^{2} \& \\
\text { Signif. Model }\end{array}$ \\
\hline (Constant) & $\mathrm{B}=5.32$ & & 0.66 & 0.51 & $\begin{array}{c}\mathrm{F}=28.37 \\
\mathrm{p}=0.000\end{array}$ \\
& $\mathrm{SE}=8.02$ & & & & \\
MAS ADI (pre) & $\mathrm{B}=0.89$ & 0.68 & 6.14 & 0.00 & Adj. $\mathrm{R}^{2}=0.62$ \\
& $\mathrm{SE}=0.15$ & & & & \\
PPT Inc. AN/CA & $\mathrm{B}=-0.11$ & -0.31 & -2.780 .01 & $\mathrm{SE}=6.36$ \\
& $\mathrm{SE}=0.04$ & & & & \\
(Constant) & $\mathrm{B}=3.68$ & & 0.45 & 0.65 & $\begin{array}{c}\mathrm{F}=26.17 \\
\mathrm{p}=0.000\end{array}$ \\
& $\mathrm{SE}=8.14$ & & & & $\begin{array}{c}\mathrm{Adj} . \mathrm{R}^{2}=0.60 \\
\mathrm{SE}=6.53\end{array}$ \\
MAS ADI (pre) & $\begin{array}{c}\mathrm{B}=0.92 \\
\mathrm{SE}=0.15\end{array}$ & 0.69 & 6.21 & 0.00 & \\
& $\mathrm{~B}=-0.09$ & -0.27 & -2.400 .02 & \\
PPT Inc. AN/HA & $\mathrm{SE}=0.04$ & & &
\end{tabular}

$\mathrm{PPT}=$ Probe Position Task; Inc. AN/CA = Incongruent Angry/Calm; Inc. AN/HA = Incongruent Angry/Happy; MAS ADI = Multidimensional Anxiety Disorders Scale for Children, Anxiety Disorders Index.

ety post-treatment. The post-treatment CBCL internalizing score was predicted by its pre-values, but not predicted significantly by PPT difference scores, although the significance of the incongruent angry versus calm difference score was at a trend level (partial correlation of $0.26 ; p=0.056)$.

Regression analyses for the ADI were repeated with the additional entry of state anxiety in the first step (a significant correlate of PPT scores), but the PPT difference scores remained significant predictors of final ADI scores indicating that state anxiety did not account for the regression results. Similarly, PPT difference scores remained significant predictors of post-treatment ADI scores after adding MASC social anxiety scores in the 
first step (a significant correlate of PPT scores).

\subsection{Changes in Relation to CBT}

Two-way repeated measures ANOVAs using time and emotion as within-subject factors revealed no significant interaction effects $(\mathrm{F}(1.35)=2.31, \mathrm{p}=0.14$ for angry/ calm; $F(1.35)=1.85, p=0.22$ for angry/happy), indicating that treatment did not affect PPT scores differentially for different emotions.

Though recognizing that there were sample size constraints, we also explored the possibility that PPT scores might change differentially with CBT in treatment responders only (response defined as a change of at least 0.5 standard deviation on the MASC ADI; $\mathrm{n}=25$ ). Analyses limited to treatment responders, however, did not show differential changes in scores in relation to CBT. Thus, we could not find evidence of differential change in PPT scores by emotion with CBT.

\section{DISCUSSION}

To summarize, clinically anxious children did not show greater attention to threat on the PPT than normal controls. However, PPT difference scores related to threat attention were correlated with self-reported social anxiety and, in anxious children, were predictive of response to CBT on the Anxiety Disorders Index of the MASC. PPT scores did not change differentially by emotion with CBT, indicating a lack of treatment-related change in attention to threat.

Lack of group differences on the PPT may relate to the benign nature of the experimental situation. State anxiety needs to be elevated for some anxious individuals to display biased attention [9], and neither group in our sample scored above the normal range for the STAIC. The STAIC was, however, correlated with incongruent PPT difference scores involving angry faces, suggesting that children who were somewhat anxious in the experimental situation exhibited higher attention to threat than those who were not. Other evidence suggests that threat perception in anxious children may be elicited more reliably with verbal rather than facial stimuli [6], and that differences in threat perception between anxious and nonanxious participants may be attenuated in young children [29].

Consistent with Waters and colleagues [13], we found an association between social anxiety and threat perception. The use of facial cues in the PPT might account for this association. Nonfacial cues might elicit other forms of threat-related biases in anxious children (e.g., depictions of dangerous situations for bias to physical threats). However, content specificity for attentional bias has not been consistently found in children [6,7].

Consistent with previous studies, attention to threat on this visual, implicit task seemed to predict limited benefits of CBT $[16,18]$. As learning is adversely affected by heightened arousal [30], threat perception (and attendant increased arousal) may interfere with learning coping strategies in CBT. Alternatively, anxious children may have difficulty successfully applying coping strategies when they are overwhelmed by perceiving many situations as threatening. These possibilities warrant further investigation.

Threat bias did not change significantly with CBT, consistent with the idea that CBT aids children in developing explicit coping strategies (likely via prefrontal mechanisms), but has limited effect on implicit attentional biases towards threat $[10,11,18]$. Such biases may need separate therapeutic attention. Bar Haim, Morag and Glickman [31] have recently developed attentional retraining programs which might be used in these vulnerable children.

Future studies could benefit from the inclusion of larger samples with greater ethnic and parental educational diversity. Replication could also be affected by the existence of several versions of the dot-probe paradigm (e.g., some with paired rather than single facial stimuli), and by debate as to the optimal time intervals to use experimentally. Most authors, however, advocate the $500 \mathrm{~ms}$ presentation of stimuli used in this study [32].

Clinically, our findings suggest potential benefit to identifying anxious children with heightened attention to threat using behavioral measures (e.g., PPT) or questionnaires (e.g., social anxiety scale of the MASC), and then targeting them for more intensive or more threatfocused intervention [17,31]. It is also possible that cognitive behavioral therapy models focused exclusively on social threat may have different effects on threat perception as measured in this study than standard CBT [33]. Further studies on the role environmental factors might play in maintaining or ameliorating threat perception, on threat perception in behaviorally inhibited children (at risk for social anxiety) [34], and on the relationship between threat perception and brain imaging, particularly in relation to treatment, are also indicated.

In conclusion, threat perception in anxious children predicted CBT outcome but did not change in response to CBT, suggesting that further studies that address this perception may offer hope to many anxious children who have yet to respond to treatment.

\section{REFERENCES}

[1] Connolly, S.D. and Bernstein, G.A. (Work Group on Quality Issues) (2007) Practice parameter for the assessment and treatment of children and adolescents with anxiety disorders. Journal of the American Academy of Child \& Adolescent Psychiatry, 46, 267-283. doi:10.1097/01.chi.0000246070.23695.06 
[2] Last, C.G., Hansen, C. and Franco, N. (1997) Anxious children in adulthood: A prospective study of adjustment. Journal of the American Academy of Child \& Adolescent Psychiatry, 36, 645-652.

doi:10.1097/00004583-199705000-00015

[3] Compton, S.N., Walkup, J.T., Albano A.M., et al. (2010) Child/adolescent anxiety multimodal study (CAMS): Rationale, design and methods. Child and Adolescent Psychiatry and Mental Health, 4, 1.

doi:10.1186/1753-2000-4-1

[4] Ginsburg, G.S., Kendall, P.C., Sakolsky, D., et al. (2011) Remission after acute treatment in children and adolescents with anxiety disorders: Findings from the CAMS. Journal of Consulting and Clinical Psychology, 79, 806813. doi:10.1037/a0025933

[5] Rynn, M., Puliafico, A., Heleniak, C., et al. (2011) Advances in pharmacotherapy for pediatric anxiety disorders. Depression and Anxiety, 28, 76-87. doi:10.1002/da.20769

[6] Puliafico, A.C. and Kendall, P.C. (2006) Threat-related attentional biases in anxious youth: A review. Clinical Child and Family Psychology Review, 9, 162-180. doi:10.1007/s10567-006-0009-x

[7] Shechner, T., Britton, J.C., Perez-Edgar, K., et al. (2012) Attention biases, anxiety, and development: Toward or away from threats or rewards? Depression and Anxiety, 29, 282-294. doi:10.1002/da.20914

[8] Bar-Haim, Y., Lamy, D., Pergamin, L., et al. (2007) Threatrelated attentional bias in anxious and nonanxious individuals: A meta-analytic study. Psychological Bulletin, 133, 1-24. doi:10.1037/0033-2909.133.1.1

[9] Rutherford, E.M., MacLeod, C. and Campbell, L.W. (2004) Negative selectivity effects and emotional selectivity effects in anxiety: Differential attentional correlates of state and trait variables. Cognition \& Emotion, 18, 711-720. doi:10.1080/02699930341000121

[10] MacLeod, C., Rutherford, E., Campbell, L., et al. (2002) Selective attention and emotional vulnerability: Assessing the causal basis of their association through the experimental manipulation of attentional bias. Journal of $A b-$ normal Psychology, 111, 107-123. doi:10.1037/0021-843X.111.1.107

[11] Browning, M., Holmes, E.A. and Harmer, C.J. (2010) The modification of attentional bias to emotional information: A review of the techniques, mechanisms, and relevance to emotional disorders. Cognitive, Affective, \& Behavioral Neuroscience, 10, 8-20. doi:10.3758/CABN.10.1.8

[12] Spector, I.P., Pecknold, J.C. and Libman, E. (2003) Selective attentional bias related to the noticeability aspect of anxiety symptoms in generalized social phobia. Journal of Anxiety Disorders, 17, 517-531. doi:10.1016/S0887-6185(02)00232-3

[13] Waters, A.M., Mogg, K., Bradley, B.P., et al. (2010) Attentional bias for emotional faces in children with generalized anxiety disorder. Journal of the American Academy of Child \& Adolescent Psychiatry, 47, 435-442. doi:10.1097/CHI.0b013e3181642992

[14] Perez-Edgar, K., Reeb-Sutherland, B.C., McDermott, J.M., et al. (2011) Attention biases to threat link behavioral in- hibition to social withdrawal over time in very young children. Journal of Abnormal Child Psychology, 39, 885-895. doi:10.1007/s10802-011-9495-5

[15] Tobon, J.I., Ouimet, A.J. and Dozois, D.J.A. (2011) Attentional bias in anxiety disorders following cognitive behavioral treatment. Journal of Cognitive Psychotherapy, 25, 114-129. doi:10.1891/0889-8391.25.2.114

[16] Legerstee, J.S., Tulen, J.H.M., Kallen, V.L., et al. (2009) Threat-related selective attention predicts treatment success in childhood anxiety disorders. Journal of the American Academy of Child \& Adolescent Psychiatry, 48, 196205. doi:10.1097/CHI.0b013e31819176e4

[17] Legerstee, J.S., Tulen, J.H.M., Dierckx, B., et al. (2010) CBT for childhood anxiety disorders: differential changes in selective attention between treatment responders and nonresponders. Journal of Child Psychology and Psychiatry, 51, 162-172. doi:10.1111/j.1469-7610.2009.02143.x

[18] Waters, A.M., Wharton, T.A., Zimmer-Gembeck, M.J., et al. (2008) Threat-based cognitive biases in anxious children before and after cognitive behavioral treatment. Behaviour Research and Therapy, 46, 358-374. doi:10.1016/j.brat.2008.01.002

[19] Mogg, K., Holmes, A., Garner, M., et al. (2008) Effects of threat cues on attentional shifting, disengagement and response slowing in anxious individuals. Behaviour Research and Therapy, 46, 656-667. doi:10.1016/j.brat.2008.02.011

[20] Manassis, K., Mendlowitz, S., Scapillato, D., et al. (2002) Group and individual cognitive behavior therapy for childhood anxiety disorders: A randomized trial. Journal of the American Academy of Child \& Adolescent Psychiatry, 41, 1423-1430. doi:10.1097/00004583-200212000-00013

[21] Mendlowitz, S., Manassis, K., Bradley, S., et al. (1999) Cognitive behavioral group treatments in childhood anxiety disorders: The role of parental involvement. Journal of the American Academy of Child \& Adolescent Psychiatry, 38, 1223-1229. doi:10.1097/00004583-199910000-00010

[22] Silverman, W.K. and Albano, A.M. (1996) The anxiety disorders interview schedule for children and parentsDSM-IV version. Graywind, New York.

[23] Achenbach, T.M. and Rescorla, L.A. (2001) Manual for the ASEBA school-age forms \& profiles. University of Vermont, Research Centre for Children, Youth \& Families, Burlington.

[24] March, J.S. (1998) Multidimensional anxiety scale for children. Multi-Health Systems, Toronto.

[25] Spielberger, C.D. (1973) Manual for the state trait anxiety inventory for children. Consulting Psychologists Press, Palo Alto.

[26] Boyle, M.H., Offord, D.R. and Racine, Y. (1993) Evaluation of the revised ontario child health study scales. Journal of Child Psychology and Psychiatry, 43, 189-213. doi:10.1111/j.1469-7610.1993.tb00979.x

[27] Tottenham, N., Tanaka, J.W., Leon, A.C., et al. (2009) The NimStim set of facial expressions: Judgments from untrained research participants. Psychiatry Research, 168, 
242-249. doi:10.1016/j.psychres.2008.05.006

[28] Manassis, K. (2008) Keys to parenting your anxious child. 2nd Edition, Barron's Educational Series, Inc., Hauppauge.

[29] Vasey, M.W. and MacLeod, C. (2001) Information processing factors in childhood anxiety: A developmental perspective. In: Vasey, M.W. and Dadds, M.R. Eds., The Developmental Psychopathology of Anxiety, Oxford University Press, New York, 253-277.

[30] Blaney, P. (1986) Affect and memory: A review. Psychological Bulletin, 99, 229-246. doi:10.1037/0033-2909.99.2.229

[31] Bar-Haim, Y., Morag, I. and Glickman, S. (2011) Training anxious children to disengage attention from threat: A randomized controlled trial. Journal of Child Psychology and Psychiatry, 52, 861-869. doi:10.1111/j.1469-7610.2011.02368.x

[32] Cooper, R.M. and Langton, S.R.H. (2006) Attentional bias to angry faces using the dot-probe task? It depends when you look for it. Behaviour Research and Therapy, 44, 1321-1329. doi:10.1016/j.brat.2005.10.004

[33] Beidel, D.C., Turner, S.M. and Morris, T.L. (2000) Behavioral treatment of childhood social phobia. Journal of Consulting and Clinical Psychology, 68, 1072-1080. doi:10.1037/0022-006X.68.6.1072

[34] Kagan, J. and Fox, N. (2006) Biology, culture, and temperamental biases. In: Eisenberg, N., Damon, W. and Lerner, R.M. Eds., Social, Emotional, and Personality Development, Handbook of Child Psychology, Wiley, New York, 167-225. 